\title{
Development of chronic pain in males with traumatic spinal cord injury: role of circulating levels of the chemokines CCL2 and CXCL10 in subacute stage
}

\author{
Laura Mordillo-Mateos ${ }^{1}$ - Antonio Sánchez-Ramos ${ }^{2}$ - Francesca Coperchini ${ }^{3}$ - Ines Bustos-Guadamillas ${ }^{1}$. \\ Carlos Alonso-Bonilla ${ }^{1}$ Eduardo Vargas-Baquero ${ }^{2} \cdot$ Inmaculada Rodriguez-Carrión $^{4} \cdot$ Mario Rotondi $^{3}$. \\ Antonio Oliviero ${ }^{1}$
}

Received: 14 October 2018 / Revised: 31 March 2019 / Accepted: 11 May 2019 / Published online: 10 June 2019

(c) The Author(s), under exclusive licence to International Spinal Cord Society 2019

\begin{abstract}
Study design Longitudinal study.

Objectives To assess the impact of spinal cord injury (SCI) on circulating levels of chemokines (CCL2 and CXCL10) and its relation with pain development.

Setting National Hospital for SCI patients.

Methods We longitudinally studied changes in the circulating levels of CCL2 and CXCL10 in 27 male patients with complete SCI who were evaluated in the early subacute phase and indeed 3 and 6 months after injury measuring at each time-point serum levels of CCL2 and CXCL10. Patients were telephonically interviewed about pain 1 year after SCI.

Results In the early subacute phase, patients with pain showed higher CXCL10 and similar CCL2 levels as opposed to those without pain. Moreover, CCL2 concentrations were positively associated with pain intensity. The results obtained by analysing the temporal profile of the chemokines suggested that CXCL10 was inclined to decrease over time, while CCL2 increased over time.

Conclusion The results of this preliminary study, the first performed in humans with traumatic SCI, suggest a link between changes in the circulating chemokine profile and pain development in subacute SCI stage as well as with severity in a more chronic stage. Large series studies will evaluate whether the circulating chemokine status can be useful as a biomarker for assessing the patients' risk for pain development.
\end{abstract}

\section{Introduction}

Pain is associated with many types of injuries of the nervous system [1]. The $60-90 \%$ of patients affected by any injury

$\triangle$ Antonio Oliviero

antonioo@ sescam.jccm.es

1 FENNSI Group, Hospital Nacional de Parapléjicos, SESCAM, Toledo, Spain

2 Sexual Medicine Unit, Rehabilitation Department, Hospital Nacional de Parapléjicos, SESCAM, Toledo, Spain

3 Unit of Internal Medicine and Endocrinology, Fondazione Salvatore Maugeri I.R.C.C.S., Laboratory for Endocrine Disruptors and University of Pavia, Pavia, Italy

4 Clinical Neurophysiology Unit, Hospital Nacional de Parapléjicos, SESCAM, Toledo, Spain suffer from chronic pain [1-4]. Spinal cord injury (SCI) associated pain is a highly debilitating chronic state resulting from damage or dysfunction of the nervous system and is often resistant to currently available treatments $[5,6]$. SCI is characterised by different phases we can summarise as follows: hyperacute phase (minutes to hours), early acute phase (days, characterised by increasing oedema, inflammation and haemorrhage), subacute phase (approximately from $48 \mathrm{~h}$ to 3 months, characterised by the starting of the repairing mechanisms), subacute towards chronic injury phase (approximately from 3 to 6 months or more) and chronic phase [7]. Taking in consideration that the phases after SCI are not linear, chronic phase typically occurs once any neurological changes stop (typically this happens between 6 and 12 months after the injury). The remodelling of the central nervous system after SCI is considered to be one of the causes of the development of SCI-associated pain. However, this debilitating condition is also associated 
to changes in the circulating levels of some inflammatory mediators that, after injury, are released from injured nerve fibres and immune cells [8-10]. These molecules can directly act on neuronal cell bodies and axons leading to peripheral sensitisation $[9,11-13]$. In recent years, accumulating studies have revealed that, among these mediators, chemokines are particularly expressed and distributed in the spinal cord under chronic pain conditions [1]. Chemokines are small proteins $(10-20 \mathrm{kDa})$ originally known from the peripheral immune system, where they orchestrate various aspects of immunity. Glia cells and neurons may produce different chemokines under conditions of neuronal stress or injury [14-16]. Indeed, chemokines are upregulated after peripheral nerve injury and contribute to the pathogenesis of neuropathic pain of peripheral nervous system origin, via different forms of neuron-glia interaction in the spinal cord. Among these chemokines CCL2 and CXCL10 raised particular attention. CCL2 is known to be released from neuronal synaptic vesicles in the spinal cord, and it is a major mediator of local inflammation and pain after peripheral nerve injury in animal models [17]. CCL2 enhances sensitivity to pain by direct action on its receptor CCR2 expressed by nociceptive and/or spinal neurons, suggesting that CCL2/CCR2-receptor signalling plays an important role in the establishment and/or persistence of pain [18]. CXCL10 is another important regulator in pain mechanism, indeed its levels were found to be elevated in spinal cord during the process of bone cancer. The activation of CXCL10/CXCR3-receptor axis is known to be involved in the development of cancer-induced bone pain demonstrating that CXCL10 could act as an important regulator in pain mechanisms [19]. Thus, in vitro and in vivo (on animal models) studies highlighted the importance of these chemokines in development of pain, however data on patients with SCI are still lacking. Recently, we assessed the impact of SCI on the spermatogenesis of patients during the early subacute stage of SCI [20]. In the present study, we used the same samples for different purpose.

The aim of our study was to describe the variation of both CCL2 and CXCL10 levels (in view to their recent evidenced role in spinal pain) during the first months after SCI. The possible relationship with the appearance of pain and these chemokine levels has been analysed.

\section{Methods}

\section{Patients}

A group of 27 male patients (mean age $34.6 \pm 8.9$ (SD), range 19-58) bearing a recent SCI were assessed. The patients were firstly evaluated in the subacute phase
( 1 month post-SCI, "T1-baseline"). The study was conducted in accordance with the Declaration of Helsinki and was approved by the institutional ethics committee. All the patients provided written informed consent. The patients included for the study had subacute traumatic SCI with a neurological level of injury between $\mathrm{C} 4$ and T12. The mean time from the injury to the first evaluation was 28 days \pm 6.1 (T1). The study was restricted to SCI patients with complete lesions (due to the fact that the samples of the present study are the same as in a previous study with different objectives [18]).They all were selected on the basis of having a grade A of the ASIA impairment scale [21] and complete absence of both somatosensory and motor evoked potentials [22]. The patient's neurological levels were cervical $(\mathrm{C} 4-\mathrm{C} 8, n=6)$ and thoracic (T1-T6, $n=7$ and T7-T12, $n=14$ ) in accordance with the International Standards for Neurological Classification of Spinal Cord Injury [22]. One year after the injury, we telephonically interviewed the patients about pain. We asked if they were suffering chronic pain, the time in which pain started and the treatment they were taking for pain and spasticity. Moreover, we asked to rate their current pain intensity using a single 11-point Numeric Rating Scale (NRS) with 0 indicating "no pain" and 10 indicating "maximal pain."

\section{CCL2 and CXCL10 chemokines ELISA assays}

Blood samples were obtained to test chemokines 1 month after injury (T1), 3 months after injury (T2) and 6 months after injury (T3). These time points were chosen arbitrarily, thus we have the possibility to depict a time course of the chemokine concentration. The concentrations of CXCL10, CCL2, in patients' serum were measured using commercially available kits (R\&D Systems). The mean minimum detectable dose of CXCL10 was $1.67 \mathrm{pg} / \mathrm{ml}$. The intra- and inter-assay coefficients of variation were $3.0 \%$ and $6.1 \%$, respectively. The mean minimum detectable dose of CCL2 was less than $5.0 \mathrm{pg} /$ $\mathrm{ml}$. The intra- and inter-assay coefficients of variation were $4.7 \%$ and $5.8 \%$, respectively. Samples were assayed in duplicate. Quality control pools for low, normal, or high concentrations were included in each assay.

\section{Data analysis}

Results are expressed as the mean and standard deviation (mean \pm standard deviation). Firstly, the patients were binary categorised based on the pain appearance (pain group or no pain group). Chemokine concentrations of the two groups were compared using Student $t$ test for unpaired data. 


\section{Chemokine time course}

Chemokine time course was evaluated in those patients in whom the three time point values were available (18 of 27) using repeated measures ANOVA (mean age 35.4 \pm 9.5 (SD), range 19-58). The data were entered into mixed model ANOVA, with CHEMOKINE (CXCL10 or CCL2 and time (T1, T2 and T3) as main factors. Follow-up ANOVAs were built in case of significant interaction. In case of significant effects, we used Tukey's test for post hoc analysis. During ANOVA execution, the degrees of freedom were corrected with Greenhouse Coefficients if sphericity could not be assumed. Results were considered significant at $p<0.05$.

\section{Individual chemokine time course}

The group analysis showed that CXCL10 tends to decrease over time (see Results), while CCL2 increased over time. For this reason, we calculated the ratio $\mathrm{T} 3 / \mathrm{T} 1$ for each patient and for each chemokine separately. The patients were then categorised in two subgroups: (1) patients that follow the group analysis (e.g. CXCL10 was lower at T3 than at T1; CCL2 was higher at T3 than at T1), and (2) patients that had a different behaviour (e.g. CXCL10 was equal or higher at T3 than at T1; CCL2 was lower at T3 than at T1). This categorisation was then used to evaluate the relationship of the individual time course of chemokines and the development of pain using a $\chi^{2}$ test.

\section{Results}

\section{Clinical data $(n=27)$}

Patient characteristics are reported in Table 1. The AIS grade A did not change in all patients throughout the study span. One year after injury, 17 out of 27 patients $(\sim 60 \%)$ referred pain (mean NRS $5.4 \pm 2.3$ ). The two groups were comparable in term of age and neurological level (age: pain: $34.3 \pm 9.7$, no pain: $35.1 \pm 7.9$, unpaired $t$ test and $p=$ 0.8259; neurological level, $\chi^{2}$ test and $p=0.7196$ ).

\section{Chemokine values 1 month after injury and its relation with pain appearance $(n=27)$}

At T1, CCL2 was similar in the patients with and without pain (no pain $445.4 \pm 171.8 \mathrm{pg} / \mathrm{ml}$, pain $488.9 \pm 152.5 \mathrm{pg} / \mathrm{ml}$, one tail, unpaired $t$ test, and $p=0.2502$; Fig. 1). At T1, CXCL10 was higher in the patients who subsequently experienced pain (no pain $44.3 \pm 17.5 \mathrm{pg} / \mathrm{ml}$, pain $62.3 \pm$ $29.6 \mathrm{pg} / \mathrm{ml}$, one tail, not assuming same variance, unpaired $t$ test, and $p=0.0291$; Fig. 1).
Chemokine values 1 month after injury and its relation with pain intensity $(n=27)$

In a second-step analysis, we compared the patients with no pain only with the patients with more intense pain (NRS $\geq$ 6). At T1, both CXCL10 and CCL2 were higher in the pain $\geq 6$ group (CXCL10: no pain, $44.3 \pm 17.5 \mathrm{pg} / \mathrm{ml}$, pain $\geq 6,71.8 \pm 36.0 \mathrm{pg} / \mathrm{ml}$, one tail, not assuming same variance, unpaired $t$ test, and $p=0.0304$; CCL2: no pain, $445.4 \pm 171.8 \mathrm{pg} / \mathrm{ml}$, pain $\geq 6,584.3 \pm 114.8 \mathrm{pg} / \mathrm{ml}$, one tail, unpaired $t$ test, and $p=0.0283$ ). No correlation was found between the individual CXCL10 and the individual NRS of patients with pain $(n=17)$ (Spearman rho $=0.3488$ and $p=0.1700$ ). On the other hand, correlation analysis between the individual CCL2 and the individual NRS of patients with pain $(n=17)$ showed that the higher the CCL2 concentration the higher the NRS (Spearman rho $=$ 0.5515 and $p=0.0217$; Fig. 2).

\section{Chemokine time course $(n=18)$}

The mean of CXCL10 levels was $39.6 \pm 16.4 \mathrm{pg} / \mathrm{ml}$ at T1, $45.8 \pm 29.8 \mathrm{pg} / \mathrm{ml}$ at $\mathrm{T} 2$ and $30.7 \pm 12.7 \mathrm{pg} / \mathrm{ml}$ at $\mathrm{T} 3$. The mean CCL2 was $471.2 \pm 161.3 \mathrm{pg} / \mathrm{ml}$ at $\mathrm{T} 1,624.9 \pm$ $250.3 \mathrm{pg} / \mathrm{ml}$ at $\mathrm{T} 2$ and $705 \pm 218.1 \mathrm{pg} / \mathrm{ml}$ at $\mathrm{T} 3$ (Fig. 3). Repeated measure ANOVA showed a significant interaction chemokine $\times$ time $\left(F_{(2,34)}=12.727\right.$ and $\left.p<0.001\right)$. Followup ANOVAs showed a significant effect of time on CCL2 $\left(F_{(2,34)}=11.150\right.$ and $\left.p<0.001\right)$ and a tendency for CXCL10 $\left(F_{(2,34)}=2.787\right.$ and $\left.p=0.0757\right)$. Post hoc analysis demonstrated a significant increment at each step time points compared to T1 for CCL2 (Tuckey honest: T1 versus $\mathrm{T} 2, p=0.0119$; and $\mathrm{T} 1$ versus $\mathrm{T} 3, p<0.001)$ and a tendency for CXCL10 to be lower at T3 (Tuckey honest: T2 versus $\mathrm{T} 3, p=0.0625$ ).

\section{Individual chemokine time course $(n=18)$}

One year after injury, pain was present in $50 \%$ of patients (9/18) of this subgroup. Unchanged or increased CXCL10 over time (T3/T1) was present in five of nine patients referring pain and in one of nine without pain $\left(\chi^{2}\right.$ test, $p=$ 0.0455). Decreased CCL2 over time (T3/T1) was present in two of nine patients referring pain and in one of nine without pain $\left(\chi^{2}\right.$ test, $\left.p=0.5271\right)$.

\section{Discussion}

This is the first prospective study longitudinally describing the time course of the serum concentrations of chemokines CCL2 and CXCL10 after complete SCI in male patients in the subacute stage. Furthermore, potential correlation with 


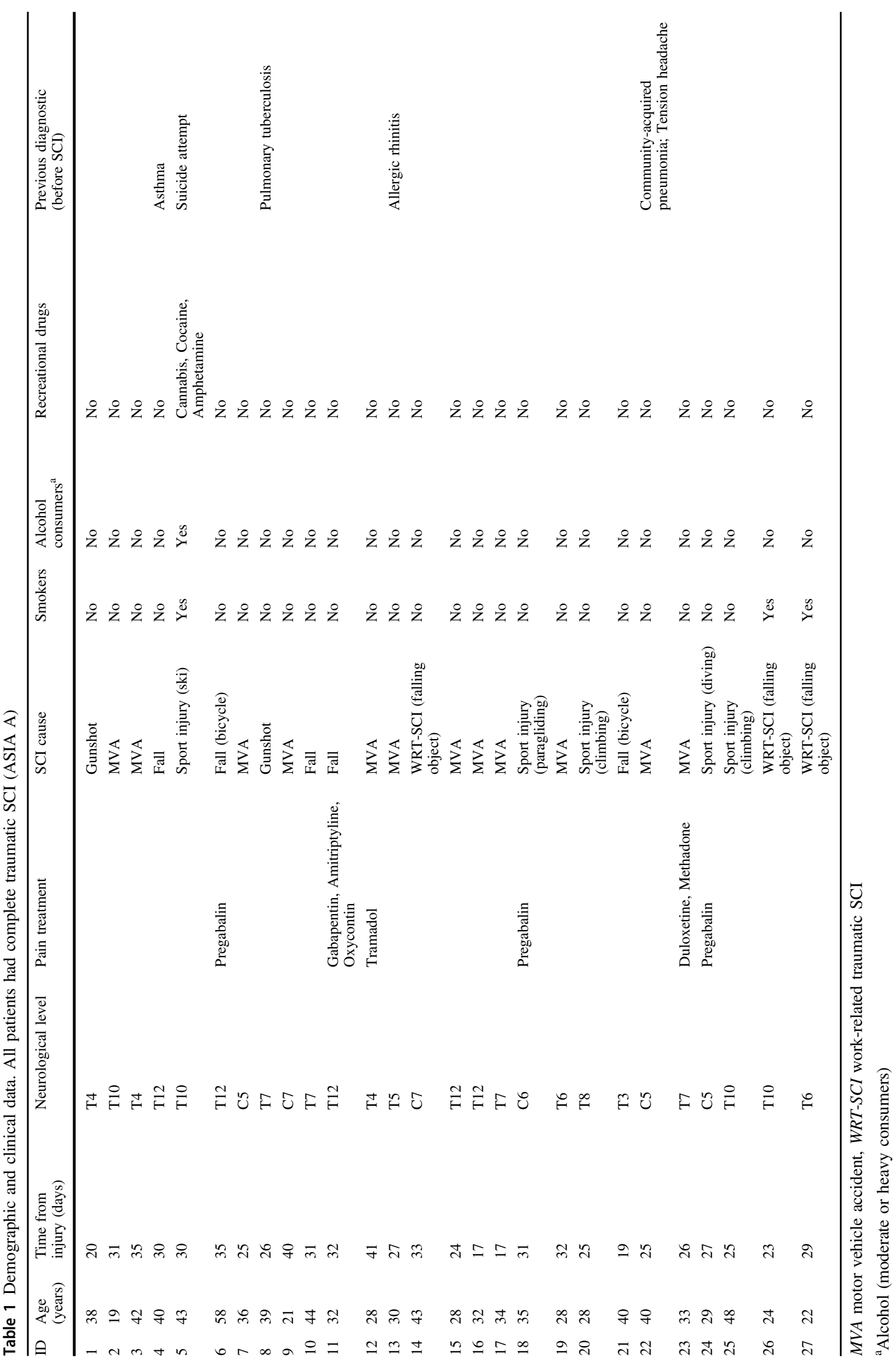


Fig. 1 Chemokine values 1 month after injury and its relation with pain appearance. Mean concentrations are expressed as $\mathrm{pg} / \mathrm{ml}$ for CxCL10 (a) and for CCL2 (b). Error bars are standard deviations. ${ }^{*} p<$ 0.05
A)

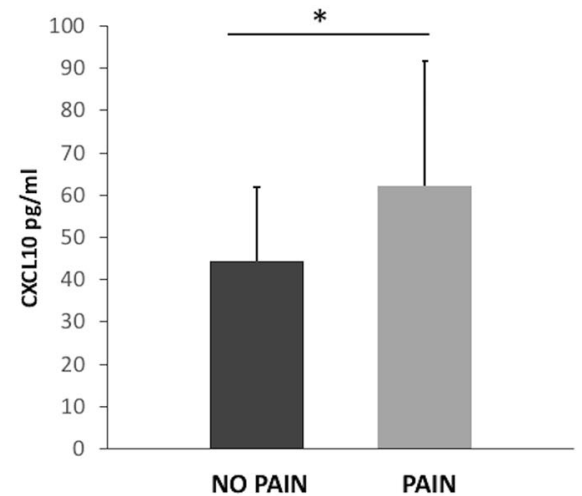

B)

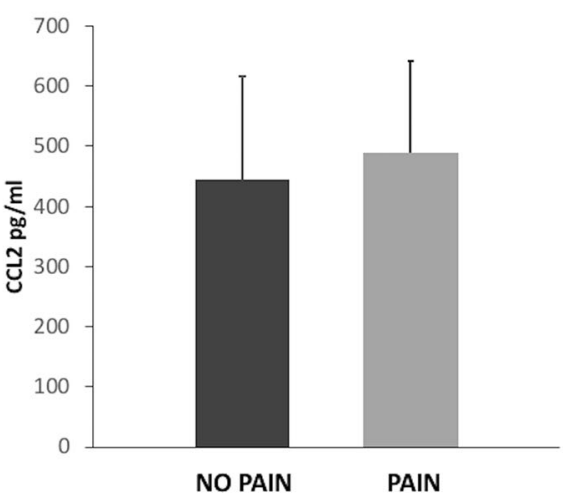

CCL2 one month after injury and its relation with pain intensity

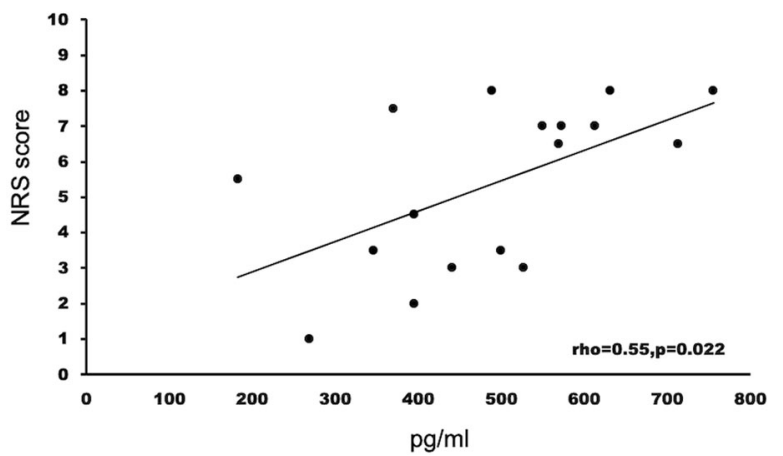

Fig. 2 Correlation between the individual CCL2 concentration 1 month after the injury and the individual NRS of patients with pain

pain appearance was established. Chemokine differences in relation to pain cannot be explained by age and neurological level differences as these variables were similar in both groups.

One year after the injury, we found that more than $60 \%$ of patients reported pain. At 1 month after injury, higher serum levels of CXCL10 were evidenced in patients reporting pain 1 year after injury in comparison to patients who did not report pain. A correlation between pain intensity 1 year after injury and the CCL2 levels was found 1 month after the injury suggesting the possibility of a role of CCL2 levels in contributing to the future pain intensity. In line with these data, patients with more intense pain (NRS $\geq 6$ ) also showed higher levels of CCL2 at the same time point compared to non-pain patients.

The results obtained by analysing the temporal profile of the chemokines suggested that CXCL10 was inclined to decrease over time, while CCL2 increased over time. The changes in the serum concentrations of CCL2 and CXCL10 overtime should be regarded as a potentially interesting finding. Indeed, several studies consistently reported that, in able-bodied individuals, no changes in the serum levels of these two chemokines are observed over time [23-25].
Briefly, Comini-Frota et al. [23] showed that in 28 ablebodied individuals the median serum CXCL10 levels did not change after 1 year of follow up. Similarly, in the study by Megel et al. [24] 29 normal-weight controls showed no changes in CCL2 levels after 3 years. Finally, Antonelli et al. [25] in 2008 reported no changes in serum levels of both CCL2 and CXCL10 in 72 able-bodied individuals over a 5 years period.

Moreover, the analysis at individual level showed that the increase of CCL2 was virtually present in all the patients evaluated. On the other hand, CXCL10 levels decreased in some patients and increased or remained unchanged in others. A final finding was that patients with unchanged or augmented levels of CXCL10 over time (in the first 6 months) had increased risk to suffer from pain 1 year after the injury.

These findings would suggest that these two chemokines seem to have a counterbalanced role in SCI-associated pain, namely: CXCL10 could participate in determining the pain appearance but not pain intensity. CCL2, conversely to CXCL10, could participate in determining the pain intensity but not the pain appearance.

We would like to underline some limitations of the present study: (1) the study was not originally designed for this purpose; (2) small sample size; (3) only male patients were included; (4) only complete lesions were included; (5) pain was only assessed through interviews over the telephone; and (6) different kind of pain were not assessed (e.g. at lesion level or below lesion level). As the findings reported were obtained from a design made for a different purpose, a future more specific study could evaluate some aspects that were not considered in the present study. For example, incomplete SCI men and women (not included in the study) may have different relationship between pain and chemokines. Moreover, to guarantee a better evaluation of pain, other more extensive pain tests should be included and more time points should be evaluated.

These limitations suggest future confirmation of the present findings that will be required by means of a 
Chemokine time course

A)

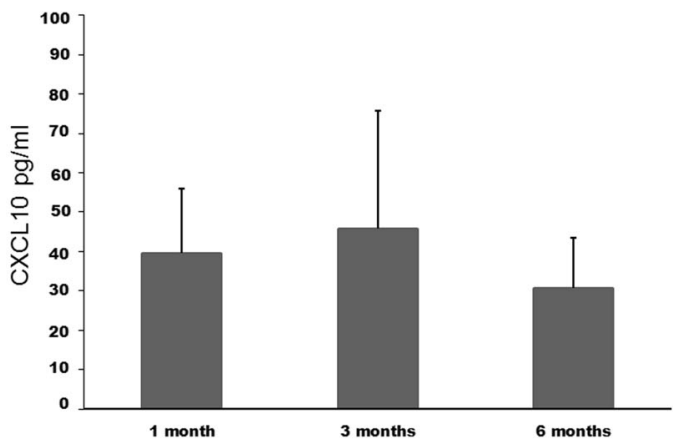

B)

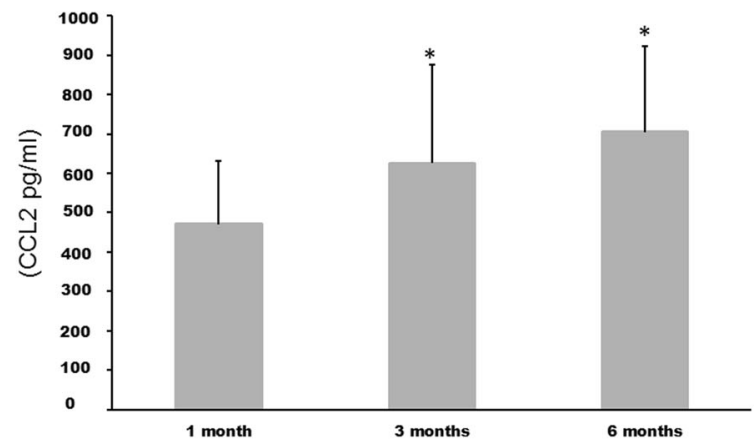

Fig. 3 Chemokine time course. Distribution of CXCL10 (a) and CCL2 (b) over time. Mean concentrations are expressed as pg/ml for CxCL10 (a) and for CCL2 (b). Error bars are standard deviations. * $p<0.05$

carefully designed longitudinal cohort studies in which the important confounders that need to be included in the analyses are identified prior to the commencement. On the other hand, the potential relevance of our results should not be underestimated because the differences in the variation of chemokine levels (at given times) in patients with SCI may help to integrate several phenomena that account for changes in pain development and severity. The variations in chemokines levels could be involved in the development or non-development of chronic pain (evidenced also in SCI animal models) in some SCI patients with similar lesion type and similar clinical picture. Confirming the literature data, the group of SCI patients we present here was extremely homogenous but only $60 \%$ developed pain [1-4].

Taken together, these results would support the concept that a better understanding of the variations of the levels of the chemokines involved in the development of neuropathic pain could be useful for the development of novel therapies [1]. Indeed, from the therapeutic point of view, neuropathic pain often proves refractory to existing therapies, but studies on the modulation of chemokine levels are giving promising results. In this view, it was demonstrated that in spinal level in rats, CCR2 antagonist blocks CCL2 nociceptive effects, suggesting that CCR 2 represents a target for the development of new therapeutic drugs for pain relief [26-28]. Furthermore, when blocking CXCL10 function in bone cancer mice with neutralising antibody, the antinociceptive effect of morphine was enhanced. Moreover, after overexpression of CXCL10 by acute injection of rmCXCL10, mice exhibited hyperalgesia immediately [29]. We suggest that, also in humans, CCL2 and CXCL10 may also have direct/indirect effects on glia and neurons that finally will be switched on a different functional state and may become pain permissive. A better understanding of the immune aspect in neuropathic pain caused by SCI could provide new ways for planning new drug-development strategies.

This is the first study in humans with traumatic SCI linking immunological changes in subacute SCI stage with pain development and severity in a more chronic stage. Clearly, these results will require confirmation but future studies should also be aimed at evaluating whether the circulating chemokine status can be useful as a biomarker allowing the individual quantification of the risk of pain development. Moreover, if causality could be proven, it will be possible to draw specific treatment to reduce the risk of pain appearance and to reduce the pain severity.

\section{Data archiving}

The datasets generated during the current study are available from the corresponding author on reasonable request.

Acknowledgements We would like to thank Rosa Maria Arriero for helpful technical help.

Funding This research was partially supported by the Grant FISCAM PI-2006/27 (Gobierno de Castilla La Mancha) and by the Department of Economy, Industry and Competitiveness and cofinanced by the European Union (FEDER) "A way to make Europe" (SAF2016-80647-R). LMM was recipient of a grant awarded by Roche.

Author contributions LMM was responsible for conducting the search, extracting and analysing data, interpreting results, updating reference lists, writing the paper and revising the final paper. ASR was responsible for recruiting and evaluating the patients. FC was responsible for screening potentially eligible studies and extracting and analysing data. $\mathrm{CAB}$ was responsible for screening potentially eligible studies and discussing results. EVB was responsible for recruiting and evaluating the patients and revising the final paper. IBG was responsible for discussing results and revising the final paper. MR was responsible for designing the study, interpreting results and updating and revising the final paper. $\mathrm{AO}$ was responsible for designing the 
study, designing the review protocol, writing the protocol, interpreting results and writing the final paper.

\section{Compliance with ethical standards}

Conflict of interest The authors declare that they have no conflict of interest.

Ethics We certify that all applicable institutional and governmental regulation concerning the ethical use of human volunteers were followed during the course of this research.

Publisher's note: Springer Nature remains neutral with regard to jurisdictional claims in published maps and institutional affiliations.

\section{References}

1. Abbadie C, Bhangoo S, De Koninck Y, Malcangio M, MelikParsadaniantz S, White FA. Chemokines and pain mechanisms. Brain Res Rev. 2009;60:125-34.

2. Bonica JJ. History of pain concepts and pain therapy. Mt Sinai J Med. 1991;58:191-202.

3. Donnelly C, Eng JJ. Pain following spinal cord injury: the impact on community reintegration. Spinal Cord. 2005;43:278-82.

4. Modirian E, Pirouzi P, Soroush M, Karbalaei-Esmaeili S, Shojaei H, Zamani H. Chronic pain after spinal cord injury: results of a long-term study. Pain Med. 2010;11:1037-43.

5. Baron R. Mechanisms of disease: neuropathic pain-a clinical perspective. Nat Clin Pract Neurol. 2006;2:95-106.

6. Von Hehn CA, Baron R, Woolf CJ. Deconstructing the neuropathic pain phenotype to reveal neural mechanisms. Neuron. 2012;73:638-52.

7. Rowland JW, Hawryluk GW, Kwon B, Fehlings MG. Current status of acute spinal cord injury pathophysiology and emerging therapies: promise on the horizon. Neurosurg Focus. 2008;25:E2.

8. Ji RR, Strichartz G. Cell signaling and the genesis of neuropathic pain. Sci STKE. 2004;252:reE14.

9. Sommer C, Kress M. Recent findings on how proinflammatory cytokines cause pain: peripheral mechanisms in inflammatory and neuropathic hyperalgesia. Neurosci Lett. 2004;361:184-7.

10. Abbadie C. Chemokines, chemokine receptors and pain. Trends Immunol. 2005;26:529-34.

11. Scholz J, Woolf CJ. Can we conquer pain? Nat Neurosci. 2002;5 (Suppl):1062-7.

12. Schafers M, Lee DH, Brors D, Yaksh TL, Sorkin LS. Increased sensitivity of injured and adjacent uninjured rat primary sensory neurons to exogenous tumor necrosis factor-alpha after spinal nerve ligation. J Neurosci. 2003;23:3028-38.

13. Kawasaki Y, Zhang L, Jen-Kun C, Ru-Rong Ji. Cytokine mechanisms of central sensitization: distinct and overlapping role of interleukin- $1 \beta$, interleukin- 6 , and tumor necrosis factor- $\alpha$ in regulating synaptic and neuronal activity in the superficial spinal cord. J Neurosci. 2008;28:5189-94.
14. de Haas AH, Boddeke HW, Biber K. Region-specific expression of immunoregulatory proteins on microglia in the healthy CNS. Glia. 2008;56:888-94.

15. Biber K, Vinet J, Boddeke HW. Neuron-microglia signaling: chemokines as versatile messengers. J Neuroimmunol. 2008;198:69-74.

16. Miller RJ, Rostene W, Apartis E, Banisadr G, Biber K, Milligan $\mathrm{ED}$, et al. Chemokine action in the nervous system. J Neurosci. 2008;28:11792-5.

17. Van Steenwinckel J, Reaux-Le Goazigo A, Pommier B, Mauborgne A, Dansereau MA, Kitabgi P, et al. CCL2 released from neuronal synaptic vesicles in the spinal cord is a major mediator of local inflammation and pain after peripheral nerve injury. J Neurosci. 2011;31:5865-75.

18. Sun JH, Yang B, Donnelly DF, Ma C, LaMotte RH. MCP-1 enhances excitability of nociceptive neurons in chronically compressed dorsal root ganglia. J Neurophysiol. 2006;96:2189-99.

19. Bu H, Shu B, Gao F, Liu C, Guan X, Ke C, et al. Spinal IFN- $\gamma$ induced protein-10 (CXCL10) mediates metastatic breast cancerinduced bone pain by activation of microglia in rat models. Breast Cancer Res Treat. 2014;143:255-63.

20. Sánchez-Ramos A, Vargas-Baquero E, Martin-de Francisco FJ, Godino-Durán JA, Rodriguez-Carrión I, Ortega-Ortega M, et al. Early spermatogenesis changes in traumatic complete spinal cordinjured adult patients. Spinal Cord. 2017;55:570-4.

21. Kirshblum S, Waring W 3rd. Updates for the international standards for neurological classification of spinal cord injury. Phys Med Rehabil Clin North Am. 2014;25:505-17.

22. Curt A, Keck ME, Dietz V. Functional outcome following spinal cord injury: significance of motor-evoked potentials and ASIA scores. Arch Phys Med Rehabil. 1998;79:81-6.

23. Comini-Frota ER, Teixeira AL, Angelo JP, Andrade MV, Brum DG, Kaimen-Maciel DR, et al. Evaluation of serum levels of chemokines during interferon- $\beta$ treatment in multiple sclerosis patients: a 1-year, observational cohort study. CNS Drugs. 2011;25:971-81.

24. Mengel E, Tillmann V, Remmel L, Kool P, Purge P, Lat E, et al. Changes in inflammatory markers in estonian pubertal boys with different BMI values and increments: a 3-year follow-up study. Obesity. 2017;25:600-7.

25. Antonelli A, Ferri C, Fallahi P, Ferrari SM, Giuggioli D, Colaci M, et al. CXCL10 (alpha) and CCL2 (beta) chemokines in systemic sclerosis-a longitudinal study. Rheumatology. 2008;47:45-9.

26. Dansereau M, Gosselin R, Pohl M, Pommier B, Mechighel P, Mauborgne A, et al. Spinal CCL2 pronociceptive action is no longer effective in CCR2 receptor antagonist-treated rats. J Neurochem. 2008;106:757-69.

27. Brodmerkel C, Huber R, Covington M, Diamond S, Hall L, Collins R, et al. Discovery and pharmacological characterization of a novel rodent-active CCR2 antagonist, INCB3344. J Immunol. 2005;175:5370-8.

28. Menetski J, Mistry S, Lu M, Mudgett J, Ransohoff R, Demartino $\mathrm{J}$, et al. Mice overexpressing chemokine ligand 2 (CCL2) in astrocytes display enhanced nociceptive responses. Neuroscience. 2007;149:706-14.

29. Ono S, Obara H, Takayanagi A, Tanabe M, Kawachi S, Itano O, et al. Suppressive effects of interleukin-18 on liver function in rat liver allografts. J Surg Res. 2012;176:293-300. 